\title{
FORENSIC MEDICAL ASSESSMENT OF MORPHOLOGICAL CHANGES AT DIFFERENT POSTMORTEM INTERVAL
}

\author{
Grygorian E., Olkhovsky V., Gubin M., Shishkin V. \\ Kharkiv National Medical University, Ukraine \\ https://doi.org/10.35339/ic.8.3.177-181
}

\begin{abstract}
Purpose: The postmortem interval (PMI) evaluation is one of priorities while performing a forensic medical examination of corpse. To date, there is lack of information on morphological postmortem changes of some internal organs. Considering the persistent need to develop the method for a precise assessment of PMI, postmortem changes in these potentially informative organs were evaluated. The aim of study was to analyze morphological postmortem changes in prostate and uterus. Materials and Methods: histological samples of 40 prostate and 40 uterine tissues $(\mathrm{n}=80)$ from corpses of deceased aged 18-75 years. Only cases with known time of death were included to study, the time of death was taken from police reports. Exclusion criteria were cases of violent death, death with massive blood loss, tumors of studied internal organs and cases when diagnosis was not made by a forensic medical examiner. The PMI of studied cases ranged from 1 to 6 days. Histological slides were made with a staining by hematoxylin and eosin, x200 magnification, using Olympus BX41 and Olympus BX46 microscopes, Olympus SC50 camera. Postmortem morphological changes were evaluated by a calculation of blank spaces percentage in microscopical structures using a JS-based software. Relationship between PMI and morphological changes was calculated by the Spearman's rank correlation. Results: the average percentage of blank spaces in uterine tissues was smaller than in prostate tissues (1.99 and 9.65, respectively). The slower growing of blank spaces was in uterus. In prostate samples, a notable increase of blank spaces was observed between 48 and 72 hours after death. After this period, the increase slowed down and then an increase was observed again between 120 and 144 hours after death. In uterine samples, a slight acceleration was observed between 72 and 120 hours after death and then slowing down between 120 and 144 hours after death. Blank spaces in evaluated histological slides were increasing directly proportional to the PMI, a statistically significant interconnection was defined $(\mathrm{p}<0.05)$. Conclusions: The morphological postmortem changes in prostate and uterus were developing at certain time frames. Blank spaces percentage, in studied histological slides, were increasing directly proportional to the PMI increase, a statistically significant interconnection was defined. Therefore, the results of study show the possibility of the evaluation of a postmortem time interval by assessing such morphological changes in these organs, which could be used in forensic medical cases.

Keywords: forensic medicine, forensic medical examination, postmortem interval, thanatology.
\end{abstract}

\section{Introduction}

Postmortem interval evaluation is still one of topical issues. Variety of approaches are being applied to define PMI, methods from different fields, such as forensic botany, genetics, entomology, biochemistry, microbiology [1-11]. But most of these methods are useful only in

Corresponding Author:

Edgar Grygorian MD, PhD student,

Department of Forensic Medicine,

Kharkiv National Medical University, Ukraine.

E-mail: 8520148@gmail.com relatively short term after death [12]. Uterus and prostate are considered to be the last internal organs to decay during human decomposition [13], so a study of these organs might be useful in cases when there was a longer time between time of death and forensic medical examination of the corpse.

\section{Purposes, Subjects and Methods}

2.1. Purpose. The aim of the study was to assess morphological changes in prostate and uterus in the postmortem period. Hypothesis was that: 1) there is a correlation between PMI and morphological changes in studied organs (uterus 
and prostate);2) postmortem changes in the uterus and prostate take different time to develop in postmortem period.

\subsection{Subjects \& Methods \\ Design}

This study was based on 40 tissue samples of prostate and 40 samples of uterus $(n=80)$ from corpses of men and women aged 18-75 years. Only cases with known time of death were included to study, the time of death was taken from police reports. Exclusion criteria were cases of violent death, cases of death with massive blood loss, tumors of studied internal organs and cases when diagnosis was not made by a forensic medical examiner. The PMI of studied cases ranged from 1 to 6 days.

The study is permitted by Ethics Committee of Kharkiv National Medical University in October 2018. The practical part of study ended by June 2021. The following attributes of the corpses were studied: age, sex, body length, fat level, scene features, presence of clothing and its peculiarities. Evaluation of postmortem changes was made using calculation of average blank spaces percentage in histological slides, in PMI starting from 1 day up to 6 days. Time of death, in studied cases, was taken from police reports on the place of death, that were presented to the examiner.

Histological slides were made with a staining by hematoxylin and eosin, x200 magnification, using Olympus BX41 and Olympus BX46 microscopes, Olympus SC50 camera.

\section{Program and Statistical Analysis}

In the histological slides studied, on the area of 250x250 pixels, the percentage of blank spaces was defined. The JS-based software was used to calculate blank spaces percentage in histological slides. The average percentages of blank spaces were calculated for each organ and for different age groups. Based on the data received, mean values and standard deviations were defined. The distribution normality was checked by Shapiro-Wilk test. Considering the nonnormality of distributions, the Spearman's rank correlation coefficients were calculated in order to detect the connections between PMI and morphological changes in studied organs. The significance level of $\mathrm{p}<0.05$ was set for all the tests. The calculations and graphs were made using StatSoft STATISTICA Version 8 (Tulsa, OK) and Microsoft Office Pro Plus 2019.

\section{Results}

The morphological changes in tissues of studied organs developed differently (Figure 1), the average percentage of blank spaces in uterus tissues was smaller than in prostate tissues (1.99 and 9.65 , respectively).

The comparison of average values of the blank spaces percentage in different postmortem interval also showed the slower growing of blank spaces in uterus than in prostate -0.08 and 0.94 , respectively at PMI of $24-48$ hours; 0.93 and 3.27 at PMI of 42-72 hours; 1.31 and 11.84 at PMI of 72-96 hours; 2.83 and 14.32 at PMI of 96120 hours; 4.79 and 17.90 at PMI of 120 144 hours (Table).

The results have been rounded to two decimal places.

In prostate samples, a notable increase of blank spaces was observed between 48 and 72 hours after death. After this period, the increase slowed down and then an increase was observed again between 120 and 144 hours after death.

In uterine samples, compared to prostate, the increase of blank spaces was less intensive, with a slight acceleration observed between 72 and 120 hours after the death and then slowing down between 120 and 144 hours after death. Blank spaces in evaluated histological slides were increasing directly proportional to the PMI, a significant interconnection $(\mathrm{p}<0.05)$ was defined. No significant differences of blank spaces percentage between age groups were observed $(\mathrm{p}<0.05)$ (Figure 2).

\section{Discussion}

The histological study of uterus showed an increase of postmortem changes over postmortem period. In cases where the duration of the postmortem period was more than 145 hours, microscopically, there were eosinophilic fibrous, dusty masses, impossible to identify. The processes of autolysis occurred more intensely and faster in the mucous membrane of the uterus, in comparison with the muscular and serous membranes, and in inner membrane of vessels, in comparison with the middle and outer membranes. The autolytic changes in the muscular membrane and vascular walls occurred more intensely in muscle fibers compared to connective tissue fibers [14].

Uddin MS et al. have shown that the morphological structure of uterus is changing over time and there is a statistically significant positive correlation between age breadth and thickness of uterus. There were changes in the morphology of uterus in relation to age [15].

According to N Abdel Rahman Mahmoud et al., light microscopic examination of prostate samples cannot detect any structure abnormality 


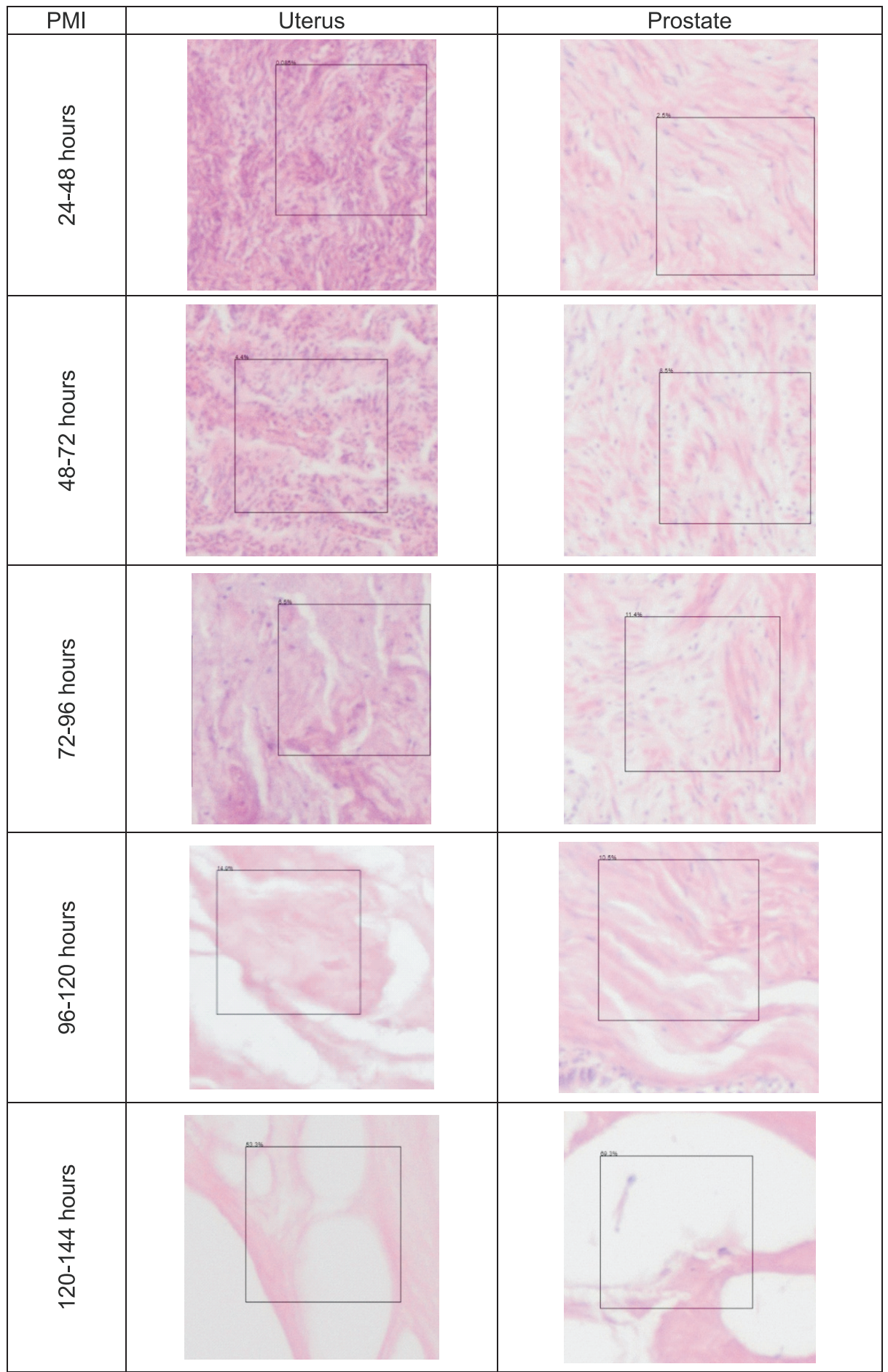

Figure 1. Histological slides of uterus and prostate tissues at different postmortem time interval Average blank spaces percentage in uterus and prostate tissues at different postmortem time interval

\begin{tabular}{|c|c|c|}
\hline Postmortem interval, hours & $\begin{array}{c}\text { Average blank spaces } \\
\text { percentage in uterus, \% }\end{array}$ & $\begin{array}{c}\text { Average blank spaces } \\
\text { percentage in prostate, \% }\end{array}$ \\
\hline $24-48$ & 0.08 & 0.94 \\
\hline $42-72$ & 0.93 & 3.27 \\
\hline $72-96$ & 1.31 & 11.84 \\
\hline $96-120$ & 2.83 & 14.32 \\
\hline $120-144$ & 4.79 & 17.90 \\
\hline Average & $\mathbf{1 . 9 9}$ & $\mathbf{9 . 6 5}$ \\
\hline
\end{tabular}




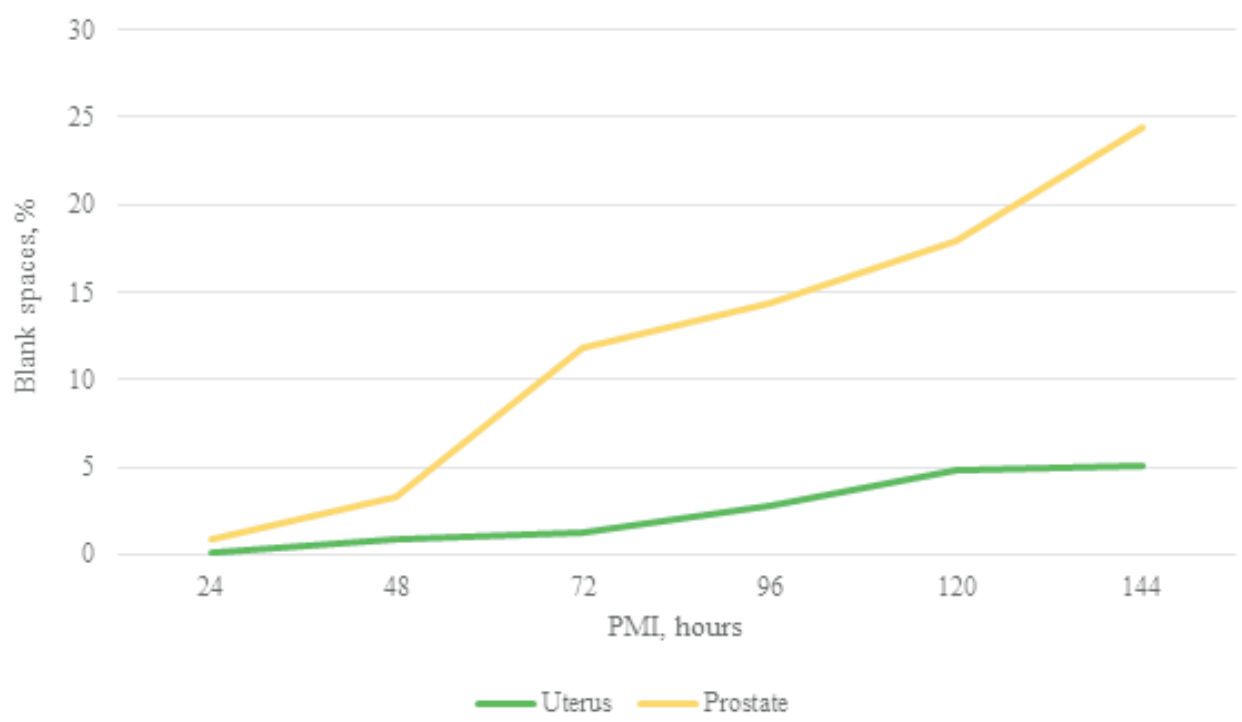

Figure 2. Blank spaces in uterus and prostate at different postmortem time interval

during the first twelve hours post mortem. In 24 hours after the death, a significant epithelial disruption, inflammatory cells and fatty degeneration began to appear in the prostatic acini. Two days PM, the prostatic acini showed significant atrophy and necrosis. By six days PM, stromal calcification started to appear. One week to four weeks PM, the prostatic acinar epithelial disruption, atrophic acini, necrosis and stromal calcification became extensive till no more normal glandular or fibromuscular architecture could be detected [16].

Elgawish, $\mathrm{R}$ et al note, that in prostate there was a normal histology at 0 and 6 hours postmortem. While, at 12 hours postmortem, most sections of the gland revealed no abnormalities except for mild focal widening between the acini. Starting from 24 hours postmortem, epithelial desquamation was seen in most acini. Seventytwo hours, obvious necrosis of prostatic acini appeared in most of samples. Degradation of collagen started and the spaces between acini became wide at 12 and 24 hours postmortem. Complete loss of stain reactivity was observed at both 60 and 72 hours postmortem. Moreover, a significant decrease in the amount of collagen stained areas in the prostate started from 12 hours postmortem. Positive immunohistochemical reaction of collagen III was detected at 0,6 and 12 hours postmortem. However, it was completely lost at 60 and 72 hours postmortem. Interestingly, the immuno-reactive area of collagen III was significantly increased at 6 hours postmortem, then a remarkable decline in immuno-reactive area was observed at 12 hours postmortem [17].

\section{Conclusion}

The morphological changes in studied organs have certain postmortem timeframes, therefore they could be used for a determination of postmortem interval. The basic hematoxylin and eosin staining could be enough to observe specific time-dependent morphological changes in histological slides. No significant differences between age groups were observed, but, assuming the quantity of cases studied, there is a need for a further study of age differences in a bigger quantity of studied cases.

\section{Declarations}

\section{Statement of Ethics}

The authors have no ethical conflicts to disclosure.

\section{Consent for publication}

All authors give their consent to publication.

\section{Disclosure Statement}

Manuscript is a part of $\mathrm{PhD}$ thesis by Edgar Grygorian

The authors report no conflicts of interest.

\section{Funding Sources}

There are no external sources of funding

\section{Data Transparency}

The data that support the findings of this study are available from the corresponding author upon reasonable request. 


\section{References}

1. Ciaffi, Romina \& Feola, Alessandro \& Perfetti, Emilio \& Manciocchi, Stefano \& Potenza, Saverio \& Marella, Gian.. Overview on the estimation of post mortem interval in forensic anthropology: review of the literature and practical experience. Romanian Journal of Legal Medicine, 26, 2018; 403-411.

2. Hauther KA, Cobaugh KL, Jantz LM, Sparer TE, DeBruyn JM. Estimating time since death from postmortem human gut microbial communities. J Forensic Sci 2015; 60 (5): 1234-1240.

3. Javan GT, Finley SJ, Can I, Wilkinson JE, Hanson JD, Tarone AM. Human thanatomicrobiome succession and time since death. Sci Rep, 2016; 6:29598.

4. Belsey SL, Flanagan RJ. Postmortem biochemistry: current applications. J Forensic Leg Med 2016; 41:49-57.

5. 20. Finley SJ, Pechal JL, Benbow ME, Robertson BK, Javan GT. Microbial Signatures of Cadaver Gravesoil During Decomposition. Microb Ecol. 2016; 71 (3): 524-529.

6. Cobaugh KL, Schaeffer SM, DeBruyn JM. Functional and Structural Succession of Soil Microbial Communities below Decomposing Human Cadavers. PLoS One. 2015; 10 (6): e0130201.

7. Bugelli V, Forni D, Bassi LA, Di Paolo M, Marra D, Lenzi S, Toni C, Giusiani M, Domenici R, Gherardi M, Vanin S. Forensic entomology and the estimation of the minimum time since death in indoor cases. J Forensic Sci. 2015; 60 (2): 525-531.

8. Mohr, R.M., Tomberlin, J.K. Development and validation of a new technique for estimating a minimum postmortem interval using adult blow fly (Diptera: Calliphoridae) carcass attendance. Int $\mathbf{J}$ Legal Med 2015; 129: 851-859.

9. Manas-Jorda S, Leon-Cortes JL, Garcia-Garcia MD, Caballero U, Infante F. Dipteran Diversity and Ecological Succession on Dead Pigs in Contrasting Mountain Habitats of Chiapas, Mexico. J Med Entomol. 2018 Jan 10;55(1):59-68.

10.Jarmusz M, Bajerlein D. Decomposition of hanging pig carcasses in a forest habitat of Poland. Forensic Sci Int. 2019 Jul;300:32-42.

11. Matuszewski S. A general approach for postmortem interval based on uniformly distributed and interconnected qualitative indicators. Int J Legal Med. 2017 May;131(3):877-884

12. Sutton L, Byrd J. An introduction to postmortem interval estimation in medicolegaldeath investigations. WIREs Forensic Sci. 2020;2:e1373

13. Holly Lutz, Alexandria Vangelatos, Neil Gottel, Antonio Osculati, Silvia Visona, Sheree J. Finley, Jack A. Gilbert, Gulnaz T. Javan. Effects of Extended Postmortem Interval on Microbial Communities in Organs of the Human Cadaver Front. Microbiol., 2020

14. Olkhovsky VO, Grygorian EK, Myroshnychenko MS, Kozlov SV, Suloiev KM, Polianskyi AO, Kaplunovskyi PA, Fedulenkova YY, Borzenkova IV. Morphological features of the uterus in women at different time intervals of the postmortem period as diagnostic criteria for establishing the postmortem interval. Wiad Lek. 2021;74(4):821-827.

15.Uddin, MS \& Al-Muhaimin, M \& Begum, N \& Sultana, Z. (2013). Age Related Changes of Human Uterus-A Postmortem Study. Medicine Today. 24. 10.3329/medtoday.v24i2.15010.

16. N Abdel Rahman Mahmoud, A Abdel Rahman Abdel Rahman Hassan, A Hassan Abdel Rahim, S Mostafa Mahmoud, O Hassan Nada, Molecular versus histopathological examination of the prostate gland in the estimation of post-mortem interval (an experimental study), QJM: An International Journal of Medicine, Volume 111, Issue suppl_1, December 2018, hcy200.054,

17.Elgawish, R., Abdelrazek, H., Desouky, A., Mohamed, R. (2021). Determination of postmortem interval through histopathological alterations and collagen evaluation in the prostate of Wistar albino rats. Zagazig Journal of Forensic Medicine, 19(2), 1-12.

Received: 03-Jul-2021

Accepted: 06-Sep-2021 